\title{
Correction to: Introduction: Trends and Developments in the Pharmaceutical and Life Sciences Industry
}

Lars Schweizer and Theodor Dingermann

\section{Correction to:}

Chapter 1 in: L. Schweizer et al. (eds.), Advances

in Pharma Business Management and Research, https://doi.org/10.1007/978-3-030-35918-8_1

The chapter was inadvertently published with a paragraph and the same has been removed from page 3 in Chap. 1 . 